\title{
A Study of the Ionic Liquid-Based Ultrasonic-Assisted Extraction of Isoliquiritigenin from Glycyrrhiza uralensis
}

\author{
Jingwei Hao, ${ }^{1,2}$ Jiahui Liu, ${ }^{2}$ Lei Zhang, ${ }^{2}$ Yunrong Jing, ${ }^{2}$ and Yubin Ji ${ }^{1}$ \\ ${ }^{1}$ Center for Life Science and Environmental Science, Harbin University of Commerce, Harbin 150076, China \\ ${ }^{2}$ College of Life Sciences and Technology, Mudanjiang Normal University, Mudanjiang 157011, China
}

Correspondence should be addressed to Yubin Ji; 120718961@qq.com

Received 4 June 2020; Revised 18 August 2020; Accepted 16 September 2020; Published 1 October 2020

Academic Editor: Beom Soo Kim

Copyright (C) 2020 Jingwei Hao et al. This is an open access article distributed under the Creative Commons Attribution License, which permits unrestricted use, distribution, and reproduction in any medium, provided the original work is properly cited.

\begin{abstract}
We successfully extracted isoliquiritigenin from Glycyrrhiza uralensis through the utilization of an ionic liquid-based ultrasonicassisted extraction (ILUAE) approach. Briefly, we utilized the solution of 1-butyl-3-methylimidazolium bromide ([BMIM]Br) as solvent and optimized key ILUAE parameters such as solid-liquid ratios, concentrations of ionic liquids, and the times of ultrasonication. Based on a single-factor experiment, we utilized the response surface method (RSM) approach to optimize the extraction procedure. The approach revealed that the optimal energy consumption time was 120 min, with the ultrasonic extraction temperature of $60^{\circ} \mathrm{C}$. Using these optimized parameters together with the solid-liquid ratio (dried G. uralensis powder: [BMIM] Br of $0.3 \mathrm{~mol} / \mathrm{L}$ ) of $1: 16.163$ and the [BMIM] $\mathrm{Br}$ of $0.3 \mathrm{~mol} / \mathrm{L}$, we achieved a $0.665 \mathrm{mg} / \mathrm{g}$ extraction yield. Overall, these findings thus indicate that we were able to effectively use ILUAE as an efficient approach to reliably extract isoliquiritigenin in a reproducible and environmentally friendly manner.
\end{abstract}

\section{Introduction}

Glycyrrhiza uralensis Fisch (sweet root, sweet grace, or Ural licorice) is a plant that is frequently used for traditional medicinal purposes in China [1-3]. Isoliquiritigenin is a flavonoid that can be extracted from the roots of G. uralensis, and that has been found to exhibit diverse pharmacological effects following its isolation from Glycyrrhiza species [4-8]. Indeed, isoliquiritigenin has been shown to be capable of inhibiting monoamine oxidase [4]. Strikingly, it has also been shown to suppress tumor cell proliferation and to promote apoptotic tumor cell death, exhibiting therapeutic efficacy 20 -fold greater than that observed for any other extracts from Glycyrrhiza when used to treat human breast cancer cells [5]. Owing to these promising properties, isoliquiritigenin has attracted substantial interest as a potential pharmaceutical agent. However, its relatively low abundance in G. uralensis and its poor solubility have made its extraction for pharmacological research challenging [9-13].

Ionic liquids are nonmolecular solvents that exhibit limited volubility, good stability, low melting point, and ability to readily dissolve certain compounds [14-18]. As such, these liquids are highly flexible and have commonly been employed in traditional Chinese medicine (TCM) as a means of reducing extraction times, improving extraction efficiency rates, and reducing the negative environmental impact associated with traditional solvents [19]. As these ionic solvents can fully penetrate medicinal raw materials, they can effectively extract the compounds of interest located therein, resulting in the observed increases in extraction rates [20-27].

Conventionally, isoliquiritigenin is extracted from $G$. uralensis via heating, boiling, or refluxing solutions containing plant materials and ethanol. However, these methods are inefficient and result in isoliquiritigenin loss, poor extraction rates, and the consumption of large organic solvent volumes.

Recently, studies have demonstrated the value of leveraging the thermal, mechanical, and cavitation properties of ultrasonication as a means of improving compound extraction via accelerating dissolution, release, and diffusion of compounds employed for TCM purposes [28-30]. Such 
ultrasonic extraction protocols can simultaneously decrease extraction time while increasing extraction rates, thus making them ideal for isolating low-abundance compounds. These approaches can also function at low temperatures and can be applied in a variety of contexts [31]. Herein, we extracted isoliquiritigenin from $G$. uralensis via an optimized approach using ionic liquids [32].

\section{Materials and Methods}

2.1. Reagents. G. uralensis was from Mudanjiang Pharmaceutical Chain Co. Ltd. while the isoliquiritigenin standard (98\% pure) was from the National Institute for the Control of Pharmaceutical and Biological Products (Beijing, China). All the ILs, namely, 1-butyl-3-methylimidazolium bromide ([BMIM]Br), 1-butyl-3-methylimidazolium chlorinate ([BMIM]Cl), 1-butyl-3-methylimidazolium hydrogen sulfate ([BMIM][HSO4]), and 1-butyl-3-methylimidazolium nitrate ([BMIM][NO3]), were from Shanghai Aichun Biotechnology Co. Ltd. (Shanghai, China). Anhydrous ethanol was from Nanjing Xingsha Chemical Co. Ltd. while methanol was from Puyang Wangda Chemical Co. Ltd. (Nanjing, China), and acetonitrile was from Jinan Century Tongda Chemical Co. Ltd. (Jinan, China). All other analytical-grade chemicals and solvents were from Beijing Chemical Reagents Co. (Beijing, China).

2.2. Analytical Conditions. For this study, we utilized a Kq$400 \mathrm{db}$ ultrasonic cleaner from Shenzhen Keweida Ultrasonic Equipment Co. Ltd. (Shenzhen, China), a 98-1-b electronic temperature-regulating heating sleeve from Heze Shengbang Instrument Development Co. Ltd. (Heze, China), a tp-213 electronic balance from Shanghai Ziyi Instrument Equipment Co. Ltd. (Shanghai, China), and a Waters 2695 HPLC instrument from Waters Co. (Milford, MA, USA). In addition, a HiQ Sil-C18 reversed-phase column $(4.6 \mathrm{~mm} \times$ $250 \mathrm{~mm}, 5 \mu \mathrm{m}$, KYA TECH Corp., Tokyo, Japan) was used for chromatographic separation.

We utilized an acetonitrile-water-acetic acid (32:68:0.5, $\mathrm{v} / \mathrm{v} / \mathrm{v})$ mobile phase for HPLC analyses, with a $1.0 \mathrm{~mL} / \mathrm{min}$ flow rate, a $10 \mu \mathrm{L}$ injection volume, and a column temperature at $25^{\circ} \mathrm{C}$ column temperature. Isoliquiritigenin was then detected based on absorbance at $350 \mathrm{~nm}$ (see Figure 1).

The resultant calibration curve used the formula ( $Y=$ $\left.3 \times 10^{7}\right)\left(X+2 \times 10^{6}\right)\left(R^{2}=0.9911, X\right.$ : isoliquiritigenin concentration, $Y$ : peak area), indicating a good linear fit for isoliquiritigenin.

2.3. Glycyrrhiza Water Content Assessment. G. uralensis was ground to produce a fine powder, with three $1 \mathrm{~g}$ portions of this powder then being weighed and transferred to an oven for storage for $24 \mathrm{~h}$, after which the absolute dry weight was calculated and used to determine moisture contents. Ultimately, Glycyrrhiza uralensis was found to have a $3.4 \%$ water content:

$$
Y=\frac{(a-b)}{a} \times 100 \% .
$$

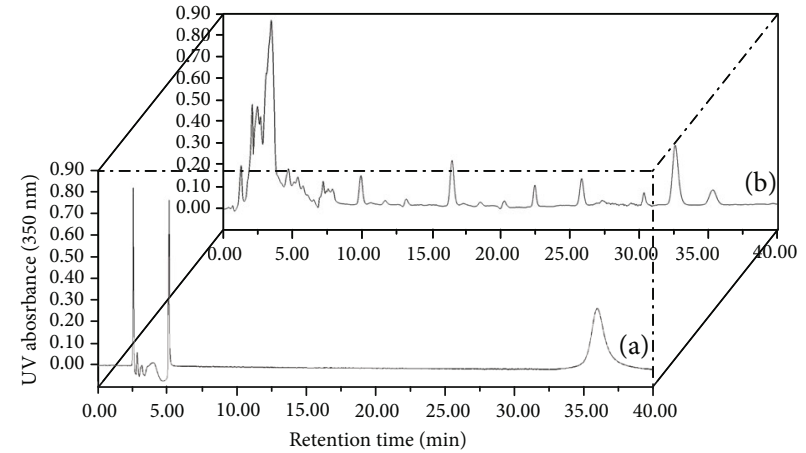

Figure 1: HPLC chromatogram for isoliquiritigenin in standard solutions (a) and sample extract (b).

Where $Y$ corresponds to water content, as a percentage, $a$ represents wet weight and $b$ represents dry weight.

2.4. Optimization of Isoliquiritigenin Extraction Using a Factorial Design. The univariate method was used to optimize the following parameters: the kinds of ionic liquids, ionic liquid concentration, solid liquid ratio $(\mathrm{g} / \mathrm{mL})$, temperature $\left({ }^{\circ} \mathrm{C}\right)$, and ultrasonic time $(\mathrm{min})$.

2.4.1. Screening of Ionic Liquids. The anion identity is considered to strongly influence the ionic liquid's properties. We utilized [BMIM]Br, [BMIM]Cl, [BMIM] $\left[\mathrm{HSO}_{4}\right]$, and $[\mathrm{BMIM}]\left[\mathrm{NO}_{3}\right]$ for these analyses. We utilized a Kq-400 db ultrasonic unit that had a $100 \mathrm{~W}$ maximum power. The following extraction parameters were utilized: $0.4 \mathrm{~g}$ of a dried sample was combined with $0.3 \mathrm{~mol} / \mathrm{L}$ of the different kinds of IL used with a solid-liquid ratio of $1: 15$ and then extracted for $120 \mathrm{~min}$ by UAE at a temperature of $60^{\circ} \mathrm{C}$. The results showed that the ionic liquids based on $\mathrm{Br}^{-}$was the most efficient of the ionic liquids tested, and [BMIM]Br was selected for subsequent experiments.

2.4.2. Effect of Concentration. The extractions were carried out in aqueous solutions with a range of [BMIM]Br IL concentrations of $(0.05,0.1,0.2,0.3,0.4,0.5$, and $0.6 \mathrm{~mol} / \mathrm{L})$, $0.4 \mathrm{~g}$ of the dried sample was combined with different [BMIM]Br IL concentrations used with a solid-liquid ratio of $1: 15$, and then extracted for $120 \mathrm{~min}$ by UAE at a temperature of $60^{\circ} \mathrm{C}$. When with the $0.3 \mathrm{~mol} / \mathrm{L}$ [BMIM] Br, the extraction rate of isoliquiritigenin was the highest. Therefore, $0.3 \mathrm{~mol} / \mathrm{L}$ [BMIM]Br was selected for subsequent experiments.

2.4.3. Effect of the Solid-Liquid Ratios. The solid-liquid ratio is an important factor because large solvent volumes could make the procedure difficult and lead to unnecessary solvent waste. By contrast, small solvent volumes may lead to incomplete extraction. A series of experiments were carried out with different solid-liquid ratios $(10,15,20,25,30$, and $40 \mathrm{~g} / \mathrm{mL}$ ) The following extraction parameters were utilized: $0.4 \mathrm{~g}$ of a dried sample was combined with the $0.3 \mathrm{~mol} / \mathrm{L}$ [BMIM]Br, the duration of ultrasonication was $120 \mathrm{~min}$, the temperature was $60^{\circ} \mathrm{C}$, and the ultrasonication power was $100 \mathrm{~W}$. Thus, the solid-liquid ratio of $1: 15$ was used in subsequent experiments. 
2.4.4. Effect of the Temperatures. The temperature is important in determining the extraction yields. Extraction temperatures of $20^{\circ} \mathrm{C}, 30^{\circ} \mathrm{C}, 40^{\circ} \mathrm{C}, 50^{\circ} \mathrm{C}, 60^{\circ} \mathrm{C}, 70^{\circ} \mathrm{C}$, and $80^{\circ} \mathrm{C}$ were investigated. The following extraction parameters were utilized: combined $0.4 \mathrm{~g}$ of a dried sample with the $0.3 \mathrm{~mol} / \mathrm{L}$ [BMIM]Br used with a solid-liquid ratio of $1: 15$, the duration of ultrasonication was $120 \mathrm{~min}$, and the ultrasonication power was $100 \mathrm{~W}$. The result showed that the extraction rate was the highest when the extraction temperature was $60^{\circ} \mathrm{C}$. Therefore, the extraction temperature of $60^{\circ} \mathrm{C}$ was selected for subsequent experiments.

2.4.5. Effect of the Ultrasonication Time. Optimization of the ultrasonication time is very important to ensure efficient extraction. Ultrasonication times of 30,60, 90, 120, 150, 180 , and $210 \mathrm{~min}$ were investigated. $0.4 \mathrm{~g}$ of accurately weighed sample was extracted with $0.3 \mathrm{~mol} / \mathrm{L}$ [BMIM]Br aqueous solutions and with a solid-liquid ratio of $1: 15$ at $60^{\circ} \mathrm{C}$, respectively. When the extraction time was $90 \mathrm{~min}$, the extraction rate of isoliquiritigenin reached the highest value.

2.5. Optimization Ultrasonic-Assisted Extraction Methods via the Response Surface Method (RSM). The RSM approach was employed to understand the interaction between optimized conditions via the use of the Box-Behnken data-processing software. Based on a single-factor investigation approach, we selected the [BMIM]Br concentration, extraction time, and solid-liquid ratio as independent variables and the isoliquiritigenin extraction rate as the dependent variable. The ultrasonic extraction temperature was fixed at $60^{\circ} \mathrm{C}$, with all other conditions then being optimized accordingly.

2.6. Assessment of ILUAE Stability and Repeatability. We evaluated the stability of this approach by dissolving isoliquiritigenin standards in [BMIM] Br via UAE using optimized assay conditions $([\mathrm{BMIM}] \mathrm{Br}=0.3 \mathrm{~mol} / \mathrm{L}$; solid-liquid ratio $=1: 16$; ultrasonic time $=120 \mathrm{~min} ; 60^{\circ} \mathrm{C}$; and $100 \mathrm{~W}$ ). Isoliquiritigenin recovery was then evaluated. Reproducibility was assessed by processing five identically weighted samples $(0.4 \mathrm{~g})$ under optimized extraction conditions.

2.7. Reference and Conventional Extraction Approaches. Two different comparisons were made to the ILUAE extraction method. In one approach, a $75 \%$ ethanol aqueous solution was used as a reference solvent for isoliquiritigenin UAE from G. uralensis. Extraction was additionally conducted under optimized extraction conditions, with solvent type being altered. Additionally, a 75\% ethanol aqueous solution was used for the reflux extraction of isoliquiritigenin with $1: 25$ solid-liquid ratio, and $80^{\circ} \mathrm{C}$ extraction temperature, and $1.5 \mathrm{~h}$ extraction duration. Then, filtrates were collected and volumes were assessed to determine extraction rates. We combined a $0.4 \mathrm{~g}$ dried sample with $10 \mathrm{~mL}$ of $75 \%$ aqueous ethanol and performed extraction for $90 \mathrm{~min}$ at $100 \mathrm{~W}$ ultrasonic power.

2.8. Statistical Analysis. To indicate the extraction efficiency of isoliquiritigenin, one-way ANOVA and Duncan's multiple range test was used to determine the significant differences

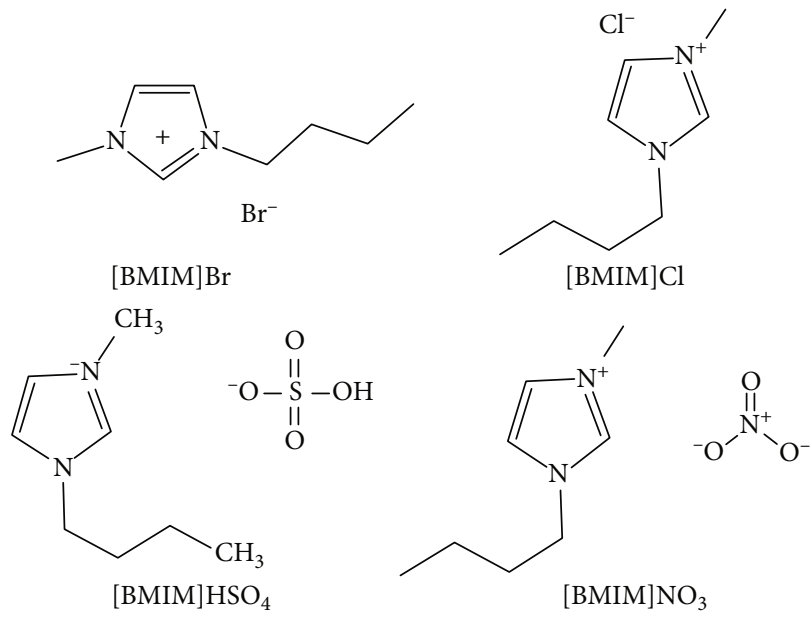

FIgURE 2: Chemical structures for ILs.

between experiments with different conditions. All statistical significance was accepted when $\alpha<0.05$. The results of the HPLC analysis were expressed as means of extraction efficiency \pm SD. Data analyses were conducted in SPSS 22.0 software.

\section{Results and Discussion}

\subsection{Single-Factor Experimental Design}

3.1.1. An Evaluation of the Impact of Different IL Solutions on UAE-Mediated Isoliquiritigenin Extraction from G. uralensis. The specific composition of a given IL ultimately determines its physical properties. For this study, we selected four different IL solutions containing different anions ([BMIM]Br, [BMIM]Cl, [BMIM] $\left[\mathrm{HSO}_{4}\right]$, and [BMIM] $\left[\mathrm{NO}_{3}\right]$; see Figure 2). Anion identity can profoundly impact the properties of these IL solutions [32]. All of these selected IL solutions were miscible with water at all concentrations. Our analyses revealed that $\mathrm{Br}^{-}$was a more efficient mediator of isoliquiritigenin extraction than any other tested solutions $(P<0.05)$. Indeed, when [BMIM]Br was added, higher isoliquiritigenin extraction rates were obtained (see Figure 3(a)). As such, [BMIM]Br was used in downstream experiments.

3.1.2. The Impact of IL Concentration on Isoliquiritigenin Extraction. We next formulated ILs at eight different concentrations $(0.05,0.1,0.2,0.3,0.4,0.5$, and $0.6 \mathrm{~mol} / \mathrm{L})$ to assess the impact of such concentrations on extraction efficiency. Additional extraction conditions were as follows: $0.4 \mathrm{~g}$ of dried sample was combined with different concentrations of ionic liquid aqueous solution at the solid-liquid ratio of $1: 15$, ultrasonication temperature of $60^{\circ} \mathrm{C}$, and $1.5 \mathrm{~h}$ ultrasonication duration. A clear dose-dependent impact of IL concentration on isoliquiritigenin yield was observed via this approach (see Figure 2(b)), with [BMIM]Br concentrations of $0.05-0.3 \mathrm{~mol} / \mathrm{L}$ being associated with rising isoliquiritigenin extraction rates that decreased significantly at higher concentrations. The results showed that there were significant differences among different concentrations $(P<0.05)$. 


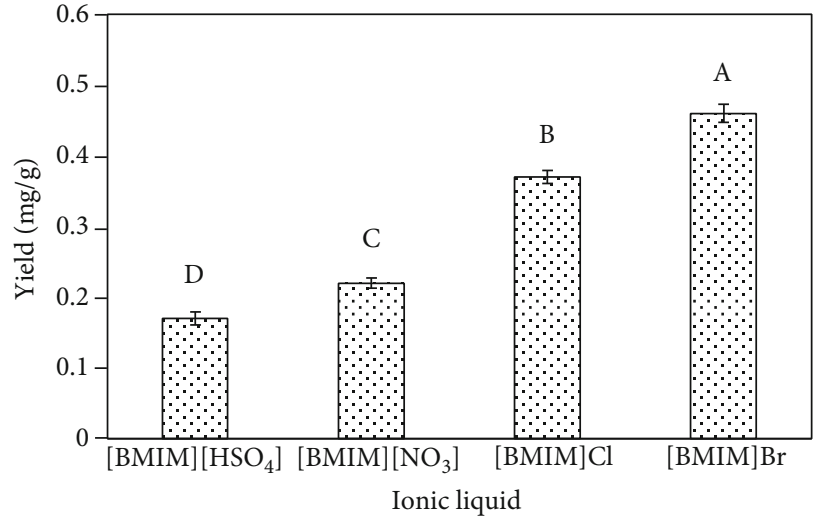

(a)

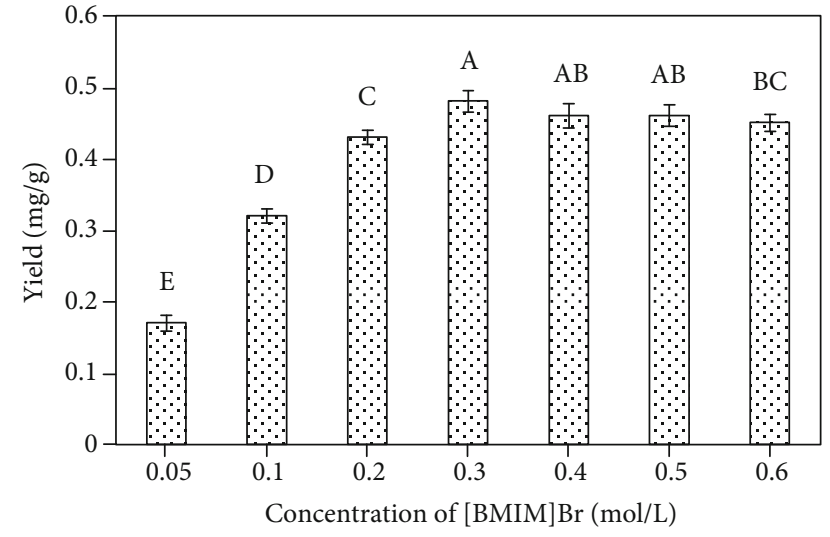

(b)

FIGURE 3: (a) The impact of IL type. The following extraction parameters were utilized: $0.4 \mathrm{~g}$ of dried sample combined with an IL concentration of $0.3 \mathrm{~mol} / \mathrm{L}$ was used with a solid-liquid ratio of $1: 15$, a $120 \mathrm{~min}$ ultrasonication duration, a temperature of $60^{\circ} \mathrm{C}$, and a $100 \mathrm{~W}$ ultrasonic power. (b) The impact of IL concentrations. The following extraction parameters were utilized: $0.4 \mathrm{~g}$ of dried sample combined with [BMIM]Br aqueous solution was used with a solid-liquid ratio of $1: 15$, a $120 \mathrm{~min}$ ultrasonication duration, a temperature of $60^{\circ} \mathrm{C}$, and a $100 \mathrm{~W}$ ultrasonic power. The different uppercase letters on the bars represent significant differences $(P<0.05)$ between treatments.

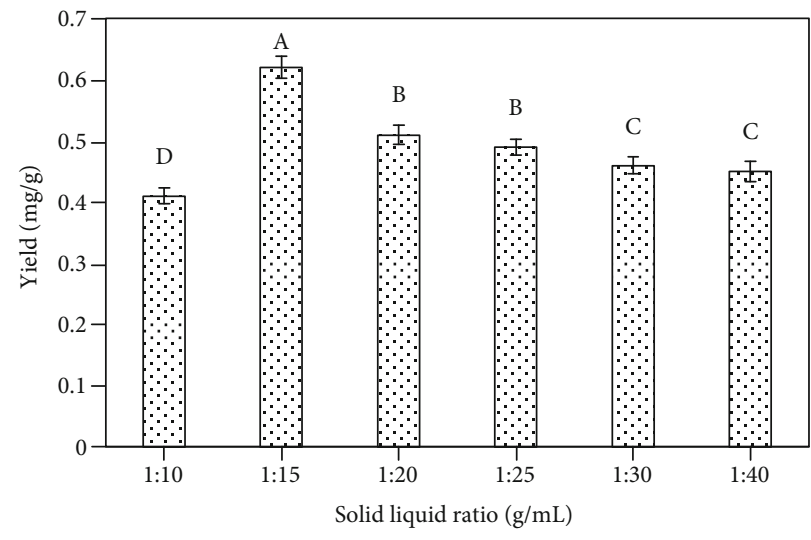

(a)

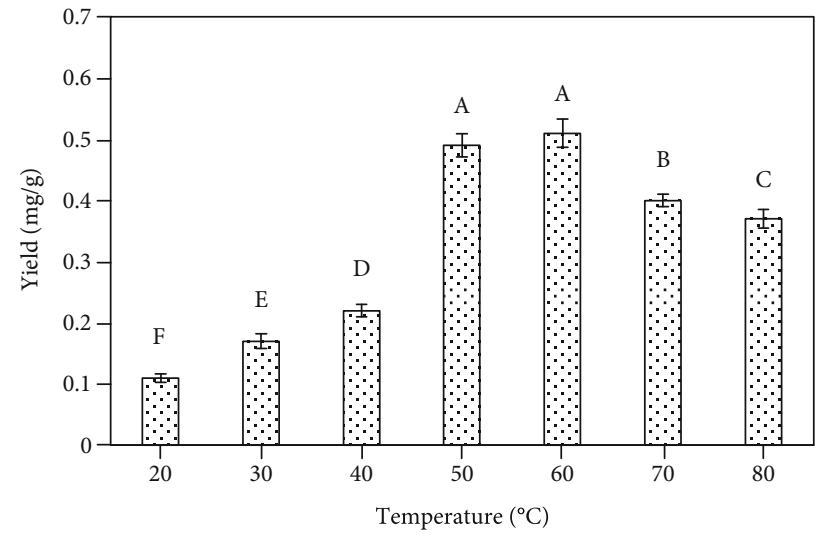

(b)

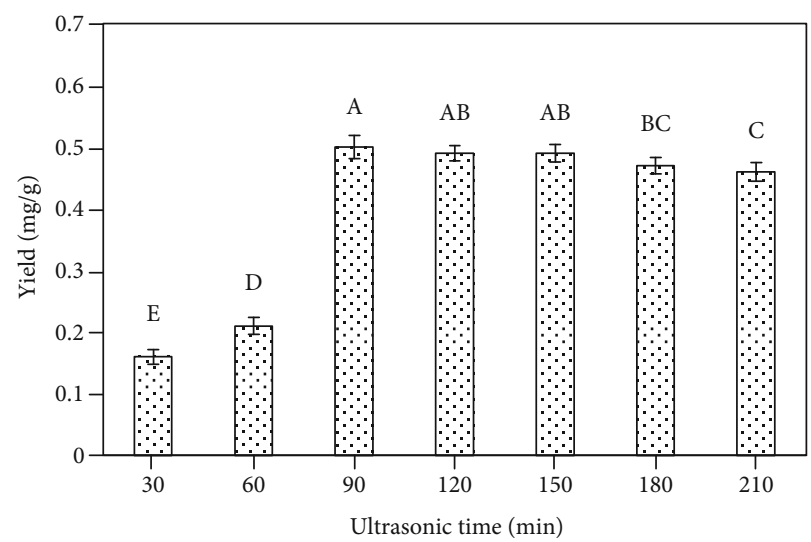

(c)

FIGURE 4: Extraction condition optimization. The following extraction parameters were utilized: (a) $0.4 \mathrm{~g}$ of a dried sample was combined with the $[\mathrm{BMIM}] \mathrm{Br}$ concentration of $0.3 \mathrm{~mol} / \mathrm{L}$, the duration of ultrasonication was $120 \mathrm{~min}$, the temperature was $60^{\circ} \mathrm{C}$, and the ultrasonication power was $100 \mathrm{~W}$. (b) $0.4 \mathrm{~g}$ of a dried sample was combined with the [BMIM]Br concentration of $0.3 \mathrm{~mol} / \mathrm{L}$, the duration of ultrasonication was $120 \mathrm{~min}$, the temperature was $60^{\circ} \mathrm{C}$, and the ultrasonication power was $100 \mathrm{~W}$. The solid-liquid ratio for this extraction was $1: 15$. (c) $0.4 \mathrm{~g}$ of a dried sample was combined with the [BMIM]Br concentration of $0.3 \mathrm{~mol} / \mathrm{L}$, the duration of ultrasonication was $30-210 \mathrm{~min}$, the temperature was $60^{\circ} \mathrm{C}$, the solid-liquid ratio was $1: 15$, and the ultrasonication power was $100 \mathrm{~W}$. The different uppercase letters on the bars represent significant differences $(P<0.05)$ between treatments. 
Given that peak extraction was achieved at an IL concentration of $0.3 \mathrm{~mol} / \mathrm{L}$, this concentration was utilized in downstream experiments. The observed dose-dependent relationship between IL concentration and extraction efficiency may suggest that higher-viscosity IL solutions may result in poorer penetration of the raw plant materials.

3.1.3. The Impact of Solid-Liquid Ratios on Isoliquiritigenin Extraction. The ratio of raw plant material to solvent can be a key determinant of extraction efficiency. Specifically, higher solvent volumes generally result in enhanced extraction efficiency, but excessively large volumes can result in complex extractions and substantial unnecessary waste.

We observed significant increases in isoliquiritigenin extraction rates when the solid-liquid ratios were between $1: 10$ and $1: 15(P<0.05)$ (see Figure $4(\mathrm{a}))$, potentially suggesting that at ratios below $1: 10$, the viscosity of the extract was too great, thereby preventing efficient isoliquiritigenin extraction. Increasing the solid-liquid ratio can improve isoliquiritigenin extraction such that peak extraction was achieved at a ratio of $1: 15$. As such, subsequent singlefactor experiments were conducted using this $1: 15$ solidliquid ratio.

3.1.4. The Impact of Different Temperatures on Isoliquiritigenin Extraction Efficiency. Temperature was found to be a key factor associated with extraction efficiency, with this efficiency rising gradually as temperature increased. There were significant differences between different temperatures $(P<0.05)$. This increase ceased at temperatures above $60^{\circ} \mathrm{C}$, after which isoliquiritigenin extraction efficiency decreased. This result may be attributable to temperature-dependent increases in extraction efficiency that are disrupted when the temperature rises too high and begins to breakdown the structure of isoliquiritigenin, resulting in an apparent decrease in extraction rate (see Figure $4(\mathrm{~b})$ ).

3.1.5. The Impact of Ultrasonication Time on Extraction Efficiency. Next, extraction was conducted across a range of extraction times $(30,60,90,120,150,180$, and $210 \mathrm{~min})$. There were significant differences between different ultrasonication times $(P<0.05)$. At extraction times $<1 \mathrm{~h}$, we observed very low isoliquiritigenin extraction rates, likely because this timeframe did not offer sufficient time for the solvent to penetrate into the root of $G$. uralensis (see Figure $4(\mathrm{c})$ ). At extraction times greater than $1.5 \mathrm{~h}$, extraction rates also tended to decrease, suggesting that prolonged extraction may result in the destruction of flavonoids such as isoliquiritigenin, leading to a lower extraction rate. Peak isoliquiritigenin extraction was thus observed after $1.5 \mathrm{~h}$, and as such this time was used for subsequent experiments.

3.2. Response Surface Methodology-Mediated Parameter Optimization. After evaluating these extraction-related parameters individually, we next explored the interactions between these factors via RSM in an effort to better optimize IL concentrations, solid-liquid ratios, and extraction time values. For this analysis, these three parameters were treated as independent variables, and the isoliquiritigenin extraction index served as dependent variable.
TABle 1: The Box-Behnken experimental design.

\begin{tabular}{lccc}
\hline Run & $\begin{array}{c}\text { Factor A } \\
\text { Concentration } \\
\text { of }[\text { BMIM]Br } \\
(\mathrm{mol} / \mathrm{L})\end{array}$ & $\begin{array}{c}\text { Factor B } \\
\text { Extraction time } \\
(\mathrm{min})\end{array}$ & $\begin{array}{c}\text { Factor C } \\
\text { Solid-liquid ratio } \\
(\mathrm{g} / \mathrm{mL})\end{array}$ \\
\hline 1 & 0.2 & 90 & $1: 15$ \\
2 & 0.2 & 90 & $1: 15$ \\
3 & 0.2 & 120 & $1: 10$ \\
4 & 0.3 & 90 & $1: 10$ \\
5 & 0.1 & 60 & $1: 15$ \\
6 & 0.2 & 60 & $1: 10$ \\
7 & 0.3 & 90 & $1: 20$ \\
8 & 0.1 & 120 & $1: 15$ \\
9 & 0.2 & 60 & $1: 20$ \\
10 & 0.2 & 90 & $1: 15$ \\
11 & 0.2 & 90 & $1: 15$ \\
12 & 0.3 & 60 & $1: 15$ \\
13 & 0.2 & 120 & $1: 20$ \\
14 & 0.1 & 90 & $1: 10$ \\
15 & 0.2 & 90 & $1: 15$ \\
16 & 0.1 & 90 & $1: 20$ \\
17 & 0.3 & 120 & $1: 15$ \\
\hline & & &
\end{tabular}

TABLE 2: The credibility analysis of the regression equations.

\begin{tabular}{lc}
\hline Index mark $^{\mathrm{a}}$ & Extraction efficiency of isoliquiritigenin \\
\hline Std. dev. & 0.025 \\
Mean & 0.44 \\
C.V.\% & 5.74 \\
PRESS & 0.028 \\
$R$-squared & 0.9799 \\
Adj $R$-squared & 0.9541 \\
Pred $R$-squared & 0.8758 \\
Adeq precision & 21.783 \\
\hline
\end{tabular}

Experimental randomization was conducted as detailed in Table 1 in an effort to maximize the impact of unexplained variability on the extraction rates. In total, we conducted 17 tests, with 5 replicates (runs 1, 2, 10, 11, and 15; see Table 1) being used to estimate the pure error sum of squares.

The predicted $R^{2}$ value of 0.8758 was reasonably consistent with the adjusted $R^{2}$ value of 0.9541 , and our precision ratio was 21.783 , indicating adequate precision (see Table 2). Furthermore, our model had very high $F$ values and low $P$ values $(P<0.0001)$ for two responses. The $F$ value of 37.95 suggests that there is only a $0.01 \%$ chance that this value is the result of noise. Indeed, any "Prob $>F$ " values of $<0.0500$ are significant, whereas values $>0.1$ are not. Based on these criteria, the following terms in our model were significant: $A, B, C, A B, A^{2}, B^{2}$, and $C^{2}$ (see Table 3 ).

The predicted $R^{2}$ value of 0.8758 was reasonably consistent with the adjusted $R^{2}$ value of 0.9541 , with the difference being less than 0.2 . The signal-to-noise ratio was measured 
TABLE 3: Test of significance for regression coefficient.

\begin{tabular}{|c|c|c|c|c|c|}
\hline Source & Sum of squares & Df & Mean square & $F$ value & $P$ value \\
\hline Model & 0.22 & 9 & 0.025 & 37.95 & $<0.0001$ \\
\hline $\mathrm{A}-[\mathrm{BMIM}] \mathrm{Br}$ & 0.048 & 1 & 0.048 & 74.25 & $<0.0001$ \\
\hline B-ultrasonic time & 0.039 & 1 & 0.039 & 60.57 & 0.0001 \\
\hline C-solid-liquid ratio & 0.026 & 1 & 0.026 & 40.87 & 0.0004 \\
\hline $\mathrm{AB}$ & $4.225 \times 10^{-3}$ & 1 & $4.225 \times 10^{-3}$ & 6.53 & 0.0378 \\
\hline $\mathrm{AC}$ & $6.250 \times 10^{-4}$ & 1 & $6.250 \times 10^{-4}$ & 0.97 & 0.3585 \\
\hline $\mathrm{BC}$ & $2.500 \times 10^{-5}$ & 1 & $2.500 \times 10^{-5}$ & 0.039 & 0.8498 \\
\hline$A^{2}$ & $3.727 \times 10^{-3}$ & 1 & $3.727 \times 10^{-3}$ & 5.76 & 0.0475 \\
\hline $\mathrm{B}^{2}$ & $5.084 \times 10^{-3}$ & 1 & $5.084 \times 10^{-3}$ & 7.86 & 0.0264 \\
\hline$C^{2}$ & 0.088 & 1 & 0.088 & 136.32 & $<0.0001$ \\
\hline Residual & $4.530 E-003$ & 7 & $6.471 \times 10^{-4}$ & & \\
\hline Lack of fit & $1.450 \times 10^{-3}$ & 3 & $4.833 \times 10^{-4}$ & 0.63 & 0.6342 \\
\hline Pure error & $3.080 \times 10^{-3}$ & 4 & $7.700 \times 10^{-4}$ & & \\
\hline Cor total & 0.23 & 16 & & & \\
\hline
\end{tabular}

by the "Adeq Precision" metric in the predictive software. As our ratio was 21.783 , which was greater than the minimum desirable level of 4 , this suggested that our signal was adequate and this model could be reliably utilized to navigate the design space.

The results of this analysis revealed that these three independent variables were related to one another based upon the second-order polynomial equation: yield $(\mathrm{mg} / \mathrm{g})=0.54+$ $0.078 \mathrm{~A}+0.07 \mathrm{~B}+0.058 \mathrm{C}+0.032 \mathrm{AB}+0.012 \mathrm{AC}-0.0025 \mathrm{BC}-$ $0.03 \mathrm{~A}^{2}-0.035 \mathrm{~B}^{2}-0.14 \mathrm{C}^{2}$.

Response surfaces corresponding to the impact of the indicated independent variables on average isoliquiritigenin extraction efficiency are shown in Figure 5, with the interactions between [BMIM]Br and solid-liquid ratio, between [BMIM]Br and ultrasonication time, and between solidliquid ratio and ultrasonication time shown in Figures 5(a), 5(b), and 5(c), respectively. Based on these analyses, the predictive software estimated that with a [BMIM]Br concentration of $0.3 \mathrm{~mol} / \mathrm{L}$, and the solid-liquid ratio of $1: 16.163$, and the ultrasonication time of $120 \mathrm{~min}$, yield could be as high as $0.665 \mathrm{mg} / \mathrm{g}$.

3.3. Verification Tests. Verification testing was conducted thrice under pint prediction RSM conditions $([\mathrm{BMIM}] \mathrm{Br}=$ $0.3 \mathrm{~mol} / \mathrm{L}$, solid-liquid ratio $=1: 16.163$, ultrasonication time $=120 \mathrm{~min}$ ). These analyses yielded an actual extraction efficiency of $0.651 \pm 0.014 \mathrm{mg} / \mathrm{g}$.

3.4. Assessment of ILUAE Stability and Repeatability. Isoliquiritigenin standard stability $(10.0$ and $20.0 \mu \mathrm{g} / \mathrm{mL})$ in $0.3 \mathrm{~mol} / \mathrm{L}$ [BMIM]Br, with a solid-liquid ratio of $1: 16$, a temperature of $60^{\circ} \mathrm{C}$, an ultrasonication duration of $120 \mathrm{~min}$, and $100 \mathrm{~W}$ power, was assessed. Average recovery rates were between $100.2 \%$ and $102.3 \%$, and no changes in retention times were observed, suggesting that this compound remains stable in a [BMIM]Br solution.
3.5. Comparison of Different Extraction Methods. We utilized two different approaches to compare the relative strengths of our ILUAE extraction approach. The extraction process was as follows: $0.4 \mathrm{~g}$ of dried samples was combined with the $75 \%$ ethanol aqueous solution, after which the solution was refluxed for $90 \mathrm{~min}$. For this approach, the yield was $0.17 \pm 0.005 \mathrm{mg} / \mathrm{g}$. The second extraction approach was identical to the strategy, but with ultrasonic extraction instead being utilized. The ultrasonication approach disrupts the external structure of raw materials at certain frequencies, enabling the solvent to better penetrate the material. The results had better extraction rates, as well as more rapid extraction. In the present study, the extraction rate was $0.28 \pm 0.009 \mathrm{mg} / \mathrm{g}$.

\section{Conclusions}

Herein, we utilized a [BMIM]Br-based UAE strategy to explore the optimal conditions for extracting isoliquiritigenin from $G$. uralensis through the use of a Box-Behnken design. Ionic liquids are promising tools for extracting active compounds from raw medicinal materials. An ultrasonicationbased extraction method was selected owing to its ability to disrupt the external structure of raw medicinal materials, facilitating enhanced solvent penetration, more rapid extraction, and a higher extraction rate. The approach can be further optimized by tuning the properties of the ionic liquids used therein. Herein, we successfully developed an ILUAE method to reliably extract isoliquiritigenin from $G$. uralensis. Briefly, we utilized a Kq-400 db ultrasonic unit (100 W maximum power), and combined $0.4 \mathrm{~g}$ of dried sample with [BMIM]Br aqueous solution. Then, we optimized IL concentrations, solid-liquid ratios, temperatures, and extraction times in order to achieve maximal extraction efficiency. To better explore how these factors were associated with one another, we utilized an RSM approach with the BoxBehnken software to further optimize these extraction 


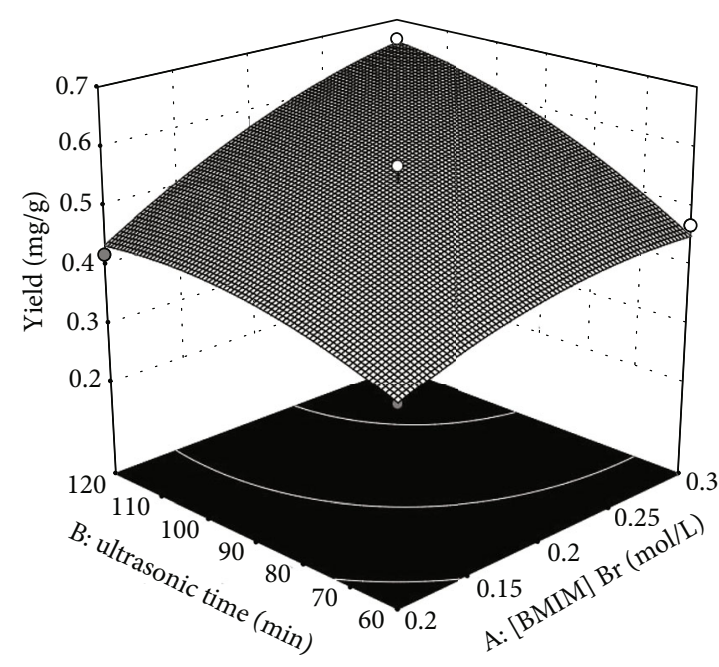

(a)

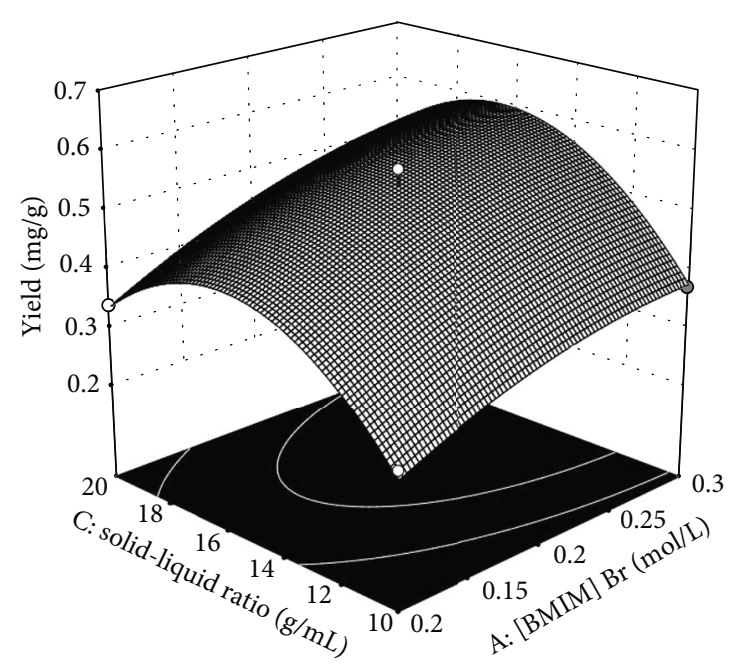

(b)

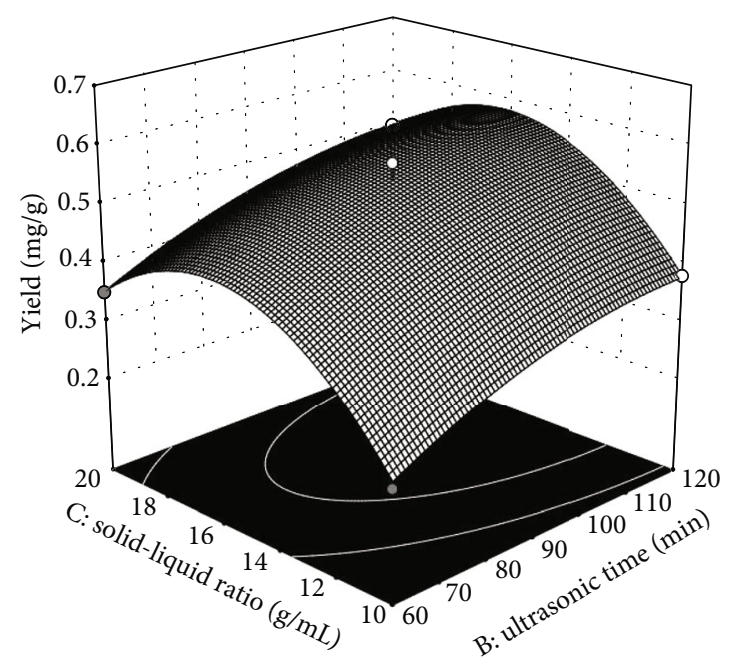

(c)

FIGURE 5: Response surface plots revealing the impact of the indicated variables on analyte extraction rates: (a) interactions between [BMIM]Br concentrations and duration of ultrasonication; (b) interactions between [BMIM]Br concentrations and solid-liquid ratio values; (c) interaction between ultrasonication duration and solid-liquid ratio.

procedures. For the analysis, ionic liquid concentrations, extraction times, and solid-liquid ratios were used as independent variables, while the rate of isoliquiritigenin was the dependent variable. Through the approach, we determined that the optimal conditions were as follows: $[\mathrm{BMIM}] \mathrm{Br}=$ $0.3 \mathrm{~mol} / \mathrm{L}$, solid-liquid ratio $=1: 16.163$, and ultrasonication time $=120 \mathrm{~min}$. Under these conditions, the maximum extraction efficiency of $0.665 \mathrm{mg} / \mathrm{g}$ could be achieved.

As a novel extraction method that combines an environmentally friendly ionic liquid with ultrasonic-assisted extraction technology, ILUAE technology not only is fast and efficient but also can be widely used for the extraction of bioactive components of TCM owing to its limited solvent and energy consumption rates. The ionic liquid-based ultrasonic-assisted extraction of isoliquiritigenin from $G$. uralensis required low amounts of energy and time, while also being able to conduct and being relatively stable. Compared with traditional reflux extraction methods that require sample heating, this approach yields high extraction efficiency while avoiding the deactivation of active compounds at high temperatures. It further overcomes other shortcomings of traditional extraction methods, such as carbonization, hydrolysis, significant organic solvent consumption, flammability, and explosion risk.

\section{Data Availability}

The data used to support the findings of this study are included within the article.

\section{Conflicts of Interest}

The authors declare that there is no conflict of interest regarding the publication of this paper. 


\section{Acknowledgments}

The authors acknowledge the financial support from the Science Research Project of the Education Department of Heilongjiang Province (number 1353MSYQN009) and Science and Technology Project of Mudanjiang (number Z2018s074).

\section{References}

[1] S. E. Quintana, C. Cueva, D. Villanueva-Bermejo, M. V. Moreno-Arribas, T. Fornari, and M. R. García-Risco, "Antioxidant and antimicrobial assessment of licorice supercritical extracts," Industrial Crops \& Products, vol. 139, article 111496, 2019.

[2] F. Moheimani, H. M. Roth, J. Cross et al., "Disruption of $\beta$ catenin/CBP signaling inhibits human airway epithelial-mesenchymal transition and repair," The International Journal of Biochemistry \& Cell Biology, vol. 68, pp. 59-69, 2015.

[3] K. Nowrin, S. S. Sohal, G. Peterson, R. Patel, and E. H. Walters, "Epithelial-mesenchymal transition as a fundamental underlying pathogenic process in COPD airways: fibrosis, remodeling and cancer," Expert Review of Respiratory Medicine, vol. 8, no. 5, pp. 547-559, 2014.

[4] S. H. He, H. G. Liu, Y. F. Zhou, and Q. F. Yue, "Liquiritin (LT) exhibits suppressive effects against the growth of human cervical cancer cells through activating caspase- 3 in vitro and xenograft mice in vivo," Biomedicine \& Pharmacotherapy, vol. 92, pp. 215-228, 2017.

[5] Y.-X. Gao, B.-F. Cheng, J.-J. Lian et al., "Liquiritin, a flavone compound from licorice, inhibits IL- $1 \beta$-induced inflammatory responses in SW982 human synovial cells," Journal of Functional Foods, vol. 33, pp. 142-148, 2017.

[6] R. Wang, C. Y. Zhang, L. P. Bai et al., "Flavonoids derived from liquorice suppress murine macrophage activation by upregulating heme oxygenase- 1 independent of Nrf2 activation," International Immunopharmacology, vol. 28, no. 2, pp. 917924, 2015.

[7] X. Ma, F. Fang, M. Song, and S. Ma, "The effect of isoliquiritigenin on learning and memory impairments induced by highfat diet via inhibiting TNF- $\alpha$ /JNK/IRS signaling," Biochemical and Biophysical Research Communicatio, vol. 464, no. 4, pp. 1090-1095, 2015.

[8] H. Gong, B. K. Zhang, M. Yan et al., "A protective mechanism of licorice (Glycyrrhiza uralensis): isoliquiritigenin stimulates detoxification system via Nrf2 activation," Journal of Ethnopharmacology, vol. 162, pp. 134-139, 2015.

[9] S. Kumar, A. Sharma, B. Madan, V. Singhal, and B. Ghosh, "Isoliquiritigenin inhibits $\mathrm{I} \kappa \mathrm{B}$ kinase activity and ROS generation to block TNF- $\alpha$ induced expression of cell adhesion molecules on human endothelial cells," Biochemical Pharmacology, vol. 73, no. 10, pp. 1602-1612, 2007.

[10] J. I. Jung, S. S. Lim, H. J. Choi et al., "Isoliquiritigenin induces apoptosis by depolarizing mitochondrial membranes in prostate cancer cells," Journal of Nutritional Biochemistry, vol. 17, no. 10, pp. 689-696, 2006.

[11] M. Kanazawa, Y. Satomi, Y. Mizutani et al., "Isoliquiritigenin inhibits the growth of prostate cancer," European Urology, vol. 43, no. 5, pp. 580-586, 2003.

[12] J. Ma, N. Y. Fu, D. B. Pang, W. Y. Wu, and A. L. Xu, “Apoptosis induced by isoliquiritigenin in human gastric cancer MGC803 cells," Planta Medica, vol. 67, no. 8, pp. 754-757, 2001.
[13] L. D. Kong, Y. Zhang, X. Pan, R. X. Tan, and C. H. K. Cheng, "Inhibition of xanthine oxidase by liquiritigenin and isoliquiritigenin isolated from Sinofranchetia chinensis," Cellular \& Molecular Life Sciences, vol. 57, no. 3, pp. 500-505, 2002.

[14] V. P. Andrade, M. Mittersteiner, M. M. Lobo et al., "A comparative study using conventional methods, ionic liquids, microwave irradiation and combinations thereof for the synthesis of 5-trifluoroacetyl-1,2,3,4-tetrahydropyridines," Tetrahedron Letters, vol. 59, no. 10, pp. 891-894, 2018.

[15] V. Rigual, T. M. Santos, J. C. Domínguez, M. V. Alonso, M. Oliet, and F. Rodriguez, "Combining autohydrolysis and ionic liquid microwave treatment to enhance enzymatic hydrolysis of Eucalyptus globulus wood," Bioresource Technology, vol. 251, pp. 197-203, 2018.

[16] S. Wahidin, A. Idris, and S. R. M. Shaleh, "Ionic liquid as a promising biobased green solvent in combination with microwave irradiation for direct biodiesel production," Bioresource Technology, vol. 206, no. 4, pp. 150-154, 2016.

[17] Y. Shen, J. K. Sun, Y. X. Yi, B. Wang, F. Xu, and R. C. Sun, "Onepot synthesis of levulinic acid from cellulose in ionic liquids," Bioresource Technology, vol. 192, no. 9, pp. 812-816, 2015.

[18] I. R. Siddiqui, A. Srivastava, S. Shamim et al., "Microwave accelerated facile and efficient synthesis of piperido[3',4':5,6] pyrano[2,3-d] pyrimidinones catalyzed by basic ionic liquid [BMIM]OH," Molecular Catalysis, vol. 382, pp. 126-135, 2014.

[19] P. Wasserscheid and W. Keim, "Ionic liquids-new "solutions" for transition metal catalysis," Angewandte Chemie International Edition, vol. 39, no. 21, pp. 3772-3789, 2000.

[20] X. Chen, X. Cao, S. Sun et al., "Hydrothermal acid hydrolysis for highly efficient separation of lignin and xylose from prehydrolysis liquor of Kraft pulping process," Separation and Purification Technology, vol. 209, pp. 741-747, 2019.

[21] A. N. Amalini, M. K. N. Haida, K. Imran, and M. K. M. Haafiz, "Relationship between dissolution temperature and properties of oil palm biomass based-regenerated cellulose films prepared via ionic liquid," Materials Chemistry and Physics, vol. 221, pp. 382-389, 2019.

[22] L. Jin, X. Yu, C. Peng et al., "Fast dissolution pretreatment of the corn Stover in gamma-valerolactone promoted by ionic liquids: selective delignification and enhanced enzymatic saccharification," Bioresource Technology, vol. 270, pp. 537-544, 2018.

[23] X. Hou, Z. Wang, J. Sun et al., "A microwave-assisted aqueous ionic liquid pretreatment to enhance enzymatic hydrolysis of Eucalyptus and its mechanism," Bioresource Technology, vol. 272, pp. 99-104, 2018.

[24] Q. Yang and X. J. Pan, "Correlation between lignin physicochemical properties and inhibition to enzymatic hydrolysis of cellulose," Biotechnology and Bioengineering, vol. 113, no. 6, pp. 1213-1224, 2016.

[25] A. R. Xu, Y. B. Zhang, W. W. Lu, K. S. Yao, and H. Xu, "Effect of alkyl chain length in anion on dissolution of cellulose in 1butyl-3-methylimidazolium carboxylate ionic liquids," Journal of Molecular Liquids, vol. 197, pp. 211-214, 2014.

[26] S. N. Sun, M. F. Li, T. Q. Yuan, F. Xu, and R. C. Sun, "Effect of ionic liquid/organic solvent pretreatment on the enzymatic hydrolysis of corncob for bioethanol production. Part 1: structural characterization of the lignins," Industrial Crops and Products, vol. 43, no. 1, pp. 570-577, 2013.

[27] J. Y. Kim, E. J. Shin, I. Y. Eom et al., "Structural features of lignin macromolecules extracted with ionic liquid from poplar 
wood," Bioresource Technology, vol. 102, no. 19, pp. 90209025, 2011.

[28] W. J. Yang, Y. Fang, J. Liang, and Q. H. Hu, "Optimization of ultrasonic extraction of Flammulina velutipes polysaccharides and evaluation of its acetylcholinesterase inhibitory activity," Food Research International, vol. 44, no. 5, pp. 1269-1275, 2011.

[29] R. A. Haddadin, "Tetralin extraction of Jordan oil shale with ultrasonic irradiation," Fuel, vol. 53, no. 3, pp. 182-185, 1974.

[30] B. Yang, Y. X. Luo, Q. J. Wu, Q. Yang, and J. Q. Kan, “Highpressure ultrasonic-assisted extraction of polysaccharides from Hovenia dulcis: extraction, structure, antioxidant activity and hypoglycemic," International Journal of Biological Macromolecules, vol. 137, pp. 676-687, 2019.

[31] X. L. Zhang and Y. H. Tan, "Modelling of ultrasonic motor with dead-zone based on Hammerstein model structure," Journal of Zhejiang University Science A, vol. 9, no. 1, pp. 5864, 2008.

[32] W. Ma, Y. Lu, R. Hu, J. Chen, Z. Zhang, and Y. Pan, “Application of ionic liquids based microwave-assisted extraction of three alkaloids $\mathrm{N}$-nornuciferine, $\mathrm{O}$-nornuciferine and nuciferine from lotus leaf," Talanta, vol. 80, no. 3, pp. 1292-1297, 2010. 